\title{
Disseminated intravascular coagulation with Fusobacterium necrophorum septicaemia
}

\author{
M.N. Potter, H.C. Drysdale, P.A. Price and A.C. Buck
}

Princess Margaret Hospital, Swindon SN1 4JU, UK.

\begin{abstract}
Summary: A 23 year old woman died within six hours of admission from acute disseminated intravascular coagulation. Fusobacterium necrophorum, a Gram negative anaerobic organism, was isolated as a single pathogen from the blood cultures. This association has not previously been reported.
\end{abstract}

\section{Introduction}

This paper reports a case of disseminated intravascular coagulation (DIC) associated with Fusobacterium necrophorum septicaemia. There follows a short review of the literature, which has shown that, in vitro, Fusobacterium species are capable of platelet aggregation and coagulation factor activation.

\section{Case report}

A 23 year old woman presented to her general practitioner with a sore throat, pyrexia and cervical lymphadenopathy. Immediately upon getting home, and before being able to take any antibiotic, she vomited, collapsed and was incontinent of faeces and urine. She was promptly transferred to hospital by another general practitioner.

There was a history of a termination of pregnancy four years previously, and pelvic inflammatory disease treated two years before admission.

On arrival at hospital she was unconscious and unresponsive to painful stimuli. She was hypotensive (blood pressure $60 / 40 \mathrm{~mm} \mathrm{Hg}$ ) with a pulse of $180 / \mathrm{min}$ and a rectal temperature of $42^{\circ} \mathrm{C}$. There was a purulent pharyngitis with small cervical lymph nodes palpable.

Initial investigations showed a haemoglobin of $13.3 \mathrm{~g} / \mathrm{dl}$, and a white cell count of $3.3 \times 10^{9} / 1$ with $33 \%$ neutrophils showing left shift and toxic

Correspondence: M.N. Potter M.A., M.R.C.P., Department of Haematology, Southmead Hospital, Bristol BS10 5NB, UK.

Accepted: 3 September 1987 changes, $54 \%$ lymphocytes and $13 \%$ monocytes. The platelet count was $82 \times 10^{9} / 1$. The Monospot test was negative. Routine biochemistry (including urea, electrolytes, glucose and amylase) was unremarkable but the arterial blood gases showed hypoxia $\left(\mathrm{PO}_{2} 4.22 \mathrm{kPa}\right)$ and a metabolic acidosis $\left(\mathrm{pH} 7.30, \mathrm{PCO}_{2} 3.59 \mathrm{kPA}\right.$, standard bicarbonate $14.3 \mathrm{mmol} / \mathrm{l})$. Chest X-ray was normal. A catheter specimen of urine showed 90 red cells $/ \mathrm{mm}^{3}$ but no white cells or organisms.

The patient was intubated and ventilated. Blood cultures were taken and gentamicin $120 \mathrm{mg}$, piperacillin $4 \mathrm{~g}$ and metronidazole $500 \mathrm{mg}$ were administered intravenously. After 2 units of a plasma expander (Haemacel) the blood pressure was $100 / 60 \mathrm{~mm} \mathrm{Hg}$ and the central venous pressure measured $+10 \mathrm{~cm}$ of $\mathrm{H}_{2} \mathrm{O}$.

Within 2 hours of admission bleeding began from venepuncture sites and all natural orifices. The haemoglobin was now $7.6 \mathrm{~g} / \mathrm{dl}$, platelets $20 \times 10^{9} / 1$, and there were no endpoints to the coagulation tests. Fibrinogen split products exceeded $320 \mathrm{U} / \mathrm{ml}$. Despite intensive support with whole blood, platelets, fresh frozen plasma and intravenous aprotinin, the patient died six hours after admission and approximately eight hours after her initial consultation with her general practitioner.

Autopsy revealed extensive haemorrhage only. There was no evidence of pelvic infection or pregnancy. Specimens taken on admission later revealed negative urine and serum toxicology screens and the aerobic blood culture was negative. However a pleomorphic Gram negative organism subsequently identified as $F$. necrophorum was isolated in pure culture after 72 hours incubation from an anaerobic Bactic tryptic soy broth bottle.

(C) The Fellowship of Postgraduate Medicine, 1988 
No pathogens were isolated on culture of urine, stool and cervical swabs.

\section{Discussion}

F. necrophorum is a Gram negative anaerobic organism which occurs as a commensal of the human oropharynx, and the gastro-intestinal and genito-urinary tracts. It is capable of causing a severe septicaemic illness, typically in young adults, with metastatic abscesses, septic pulmonary emboli, septic arthritis, osteomyelitis and acute liver dysfunction. ${ }^{1,2,3}$ Most patients with $F$. necrophorum septicaemia have an antecedent history of a sore throat, ${ }^{1,2,4}$ although in females infection may originate in the pelvis. Sometimes the primary source of infection is not evident. ${ }^{3}$

Henry et $a l .{ }^{5}$ describe 26 patients with a positive blood culture for $F$. necrophorum over a period of 5 years at the Boston City Hospital. This represented $0.9 \%$ of all bacteraemic patients during this period at that institution, but our experience is that $F$. necrophorum is isolated much less frequently from blood cultures.

The reported haematological complications of $F$. necrophorum infection are limited to suppurative thrombophlebitis of the internal jugular vein as a result of local spread from the oropharynx. ${ }^{2,4}$ In one case $^{3}$ an abnormal prothrombin time and partial thromboplastin time is mentioned. This occurred in a patient with acute hepatic dysfunction, and the authors state that the fibrinogen and fibrin split product levels were normal. (No mention is made of the platelet count). We do not believe, therefore, that this was a case of consumption coagulopathy.

\section{References}

1. Moor-Gillon, J., Lee, T.H., Eykyn, S.J. \& Phillips, I. Necrobacillosis: a forgotten disease. $\mathrm{Br}$ Med $J$ 1984, 288: $1526-1527$.

2. Seidenfeld, S.M., Sutker, W.L. \& Luby, J.P. Fusobacterium necrophorum septicemia following oropharyngeal infection. JAMA 1982, 248: 1348-1350.

3. Bouvier, D.P., Armstrong, R.D., Bradshaw, D.M. \& Hoenzsch, R.E. Fusobacterium necrophorum septicaemia without a primary focus. South Med J 1985, 78: 15211522.

4. Sanders, R.V., Kirkpatrick, M.B., Dacso, C.C. \& Bass, J.B. Suppurative thrombophlebitis of the internal jugular vein. Ala J Med Sci 1986, 23: 92-95.

5. Henry, S., De Mari, A. \& McCabe, W.R. Bacteremia due to Fusobacterium species. Am J Med 1983, 75: 225-231.
Laboratory work has shown that $F$. necrophorum has a classical lipopolysaccharide endotoxin (LPS) and that the virulence of the organism is related to this cell wall structure, in particular to the lipid A component. ${ }^{6}$

Forrester et al. ${ }^{7}$ have demonstrated that virulent strains of $F$. necrophorum are capable of the in vitro aggregation of human platelets in platelet rich plasma. Interestingly, none of the avirulent strains were capable of this reaction. This aggregation was found to be dependent upon the presence of plasma factor(s), since gel-filtered platelets were less sensitive to aggregation. Their sensitivity was fully restored by the addition of plasma, and partially restored by fibrinogen.

Bjornson ${ }^{8}$ gives evidence that the LPS of Gram negative anaerobes, including Fusobacterium species, is capable of the in vitro activation of the coagulation cascade. Further ${ }^{9}$ he shows that such LPS is capable of activating purified human Hageman factor (Clotting factor XII) - as measured by the generation of kallikrein from prekallikrein. Hageman factor activation is the initiating step of the intrinsic coagulation pathway.

These experimental observations provide a possible pathophysiological basis of a consumption coagulopathy and, together with our case report, suggest that DIC be added to the list of complications of $F$. necrophorum septicaemia.

\section{Acknowledgements}

We are grateful for the assistance given by the PHLS Anaerobic Reference Unit with the identification of our isolate and to Miss H.L. Spurrier ALA for her help in searching the literature.

6. Garcia, M.M., Charlton, K.M. \& McKay, K.A. Characterization of endotoxin from Fusobacterium necrophorum. Infect Immun 1975, 11: 371-379.

7. Forrester, L.J., Campbell, B.J., Berg, J.N. \& Barrett, J.T. Aggregation of platelets by Fusobacterium necrophorum. J Clin Microbiol 1985, 22: 245-249.

8. Bjornson, H.S. \& Hill, E.O. Bacteroidaceae in thromboembolic disease: effects of cell wall components on blood coagulation in vivo and in vitro. Infect Immun 1973, 8: 911-918.

9. Bjornson, H.S. Activation of Hageman factor by lipopolysaccharides of Bacteroides fragilis, Bacteroides vulgatus and Fusobacterium mortiferum. Rev Infect Dis 1984, 6 (Suppl. 1): 30-33. 